\title{
The interaction between permethrin exposure and malaria infection affects the host-seeking behaviour of mosquitoes
}

\author{
Kevin Thiévent ${ }^{*}$ (D), Gaël Hauser, Obada Elaian and Jacob C. Koella
}

\begin{abstract}
Background: Insecticide-treated bed nets (ITNs) help to control malaria by mechanically impeding the biting of mosquitoes, by repelling and irritating them and by killing them. In contrast to spatial repellency, irritancy implies that mosquitoes contact the ITN and are exposed to at least a sub-lethal dose of insecticide, which impedes their further blood-seeking. This would weaken the transmission of malaria, if mosquitoes are infectious.

Methods: It was therefore tested whether sub-lethal exposure to permethrin impedes blood-feeding differently in uninfected mosquitoes and in mosquitoes carrying the non-transmissible stage (oocysts) or the infectious stage (sporozoites) of the malaria parasite Plasmodium berghei. In addition, as the degree of irritancy determines the dose of insecticide the mosquitoes may receive, the irritancy to permethrin of infected and uninfected mosquitoes was compared.

Results: In this laboratory setting, sub-lethal exposure to permethrin inhibited the blood-seeking behaviour of Anopheles gambiae mosquitoes for almost $48 \mathrm{~h}$. Although infection by malaria did not affect the irritancy of the mosquitoes to permethrin at either the developmental stage or the infectious stage, both stages of infection shortened the duration of inhibition of blood-seeking.

Conclusions: The results suggest that the impact of ITNs may be weaker for malaria-infected than for uninfected mosquitoes. This will help to understand the global impact of ITNs on the transmission of malaria and gives a more complete picture of the effectiveness of that vector control measure.
\end{abstract}

Keywords: Insecticide-treated bed nets (ITNs), Irritancy, Sub-lethal effects, Malaria infection, Host-seeking behaviour

\section{Background}

In the last few decades, intensifying malaria control with insecticides that target adult mosquitoes, in particular insecticide-treated bed nets (ITNs), has decreased drastically the burden of malaria [1]. The efficacy of ITNs is partly due to their dual mode of protection: they protect the community by killing mosquitoes, thereby lowering the likelihood that mosquitoes live long enough to transmit malaria, and they protect individuals with the physical barrier of the net and the insecticide's repellency to

*Correspondence: kevin.thievent@gmail.com

Institute of Biology, University of Neuchâtel, Rue Emile-Argand 11,

2000 Neuchâtel, Switzerland mosquitoes, thereby reducing the risk that users become infected [2].

The repellency of insecticides has two components: spatial repellency and contact irritancy [3]. Spatial repellency occurs when mosquitoes detect the volatiles of the insecticide and then avoid the area near the ITN. It thus occurs before mosquitoes have had any physical contact with the ITN. Irritancy occurs when the physical contact of the mosquitoes with the insecticide makes them fly away. The relative importance of the two mechanisms of repellency depends on the insecticide. Permethrin, for example, is only slightly spatially repellent but strongly irritant, whereas DTT repels and irritates mosquitoes [3-5].

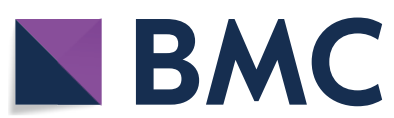

(C) The Author(s) 2019. This article is distributed under the terms of the Creative Commons Attribution 4.0 International License (http://creativecommons.org/licenses/by/4.0/), which permits unrestricted use, distribution, and reproduction in any medium, provided you give appropriate credit to the original author(s) and the source, provide a link to the Creative Commons license, and indicate if changes were made. The Creative Commons Public Domain Dedication waiver (http://creativecommons.org/ publicdomain/zero/1.0/) applies to the data made available in this article, unless otherwise stated. 
Since irritancy implies that mosquitoes contact the insecticide, and thus are exposed to at least a small (sublethal) dose of the insecticide, one of the differences between irritancy and spatial repellency is that the former may further affect the mosquitoes' physiology or behaviour [6-8].

Most studies of sub-lethal effects on mosquitoes deal with larval exposure to pesticides and its effects on lifehistory traits (reproductive success, adult longevity, sex ratio [9-13]) and on vector competence, e.g., arboviruses [14-16] and malaria [17]. While sub-lethal exposure of adults has been less well studied, a general feature of exposure to insecticides, in particular pyrethroids, appears to be that it reduces the feeding motivation for some time after exposure. This has been observed in tsetse flies (Glossina spp.) [18, 19], spider mites (Tetranychus urticae) [20], Drosophila melanogaster [21], Aedes aegypti [22], and in several species of Anopheles [23]. Sub-lethal exposure to pyrethroids also reduces the time of activation to flight and alters the flight direction of mosquitoes Culex quinquefasciatus, Ae. aegypti and $A$. albimanus [24]. These effects probably impede the subsequent search for hosts and may explain that the presence of ITNs reduces the number of mosquitoes that re-enter a house immediately after having exited it [25]. Overall, it thus appears that irritant pyrethroid insecticides can protect users of ITNs from being bitten and reduce the feeding motivation of mosquitoes to protect non-users.

Both of these aspects of protection have the greatest impact on malaria transmission when mosquitoes carry sporozoites, since both irritancy and feeding inhibition will then keep people from being infected. Unfortunately, since sporozoites make mosquitoes more motivated to bite [26-30], it could be expected that the insecticide is less protective against infectious mosquitoes. Indeed, in a previous experiment infectious mosquitoes (carrying sporozoites) were less repelled by ITNs than uninfected ones [31]. But how malaria infection affects the irritancy or feeding inhibition caused by pyrethroid insecticides is unknown.

Therefore, with this study, it was investigated whether infection of the mosquito A. gambiae by malaria affects the irritancy and the feeding inhibition induced by permethrin, a pyrethroid insecticide. In particular, it was tested whether mosquitoes infected with the non-infectious oocyst stage or the infectious sporozoite-stage of the rodent malaria parasite, Plasmodium berghei, are more or less inhibited by sub-lethal exposures to permethrin than uninfected mosquitoes. Although mosquitoes are increasingly resistant against pyrethroids, the experiment was done with the insecticide-sensitive Kisumu colony of A. gambiae. The experiment thus gives a first impression of the impact of an insecticide on the feeding behaviour of malaria-infected mosquitoes, and is not an attempt to predict the situation in the field. In particular we do not consider the long-term impact of sub-lethal exposure to an insecticide in resistant mosquitos, which may include impeded parasite development and transmission and cumulative reduction of mosquito survival over time [32]. Our experiment was done with permethrin because of its use on several commercially available ITNs (e.g. Olyset ${ }^{\circledR}$ and Olyset ${ }^{\circledR}$ Plus nets (Sumitomo Chemical Co. Ltd., Tokyo, Japan) [33]).

\section{Methods \\ Mosquito and parasite}

The experiment was done with the Kisumu colony of $A$. gambiae from Western Kenya [34] and with a strain of the rodent malaria parasite $P$. berghei ANKA expressing green fluorescent protein (GFP). Professor Heussler from the University of Bern kindly provided mice infected with gametocytes to infect the mosquitoes.

\section{Mosquito rearing and infection}

The tests for irritancy and for sub-lethal effects on feeding motivation were done in two separate experiments. For irritancy 600 mosquito larvae were reared individually in 12-well-plates in $3 \mathrm{ml}$ of de-ionized water, while for the sub-lethal effects 1200 larvae were reared in groups of 200 in trays $(35 \times 21 \times 4 \mathrm{~cm})$ containing $1 \mathrm{l}$ of deionized water. In both experiments, larvae were reared with a standard level of fish food [34] (day of hatching: $0.04 \mathrm{mg}$ of Tetramin fish food per larva; day $1 \mathrm{after}$ hatching: $0.06 \mathrm{mg}$; age 2: $0.08 \mathrm{mg}$; age $3: 0.16 \mathrm{mg}$; age 4 : $0.32 \mathrm{mg}$; age 5 and more: $0.6 \mathrm{mg}$ ), pupae were haphazardly moved to cages $(21 \times 21 \times 21 \mathrm{~cm})$ and the males were removed at most $24 \mathrm{~h}$ after emergence so that the females were virgin. The females were first maintained at $26 \pm 1{ }^{\circ} \mathrm{C}$ and $70 \pm 5 \%$ humidity. One day before infection, the temperature was decreased by $2{ }^{\circ} \mathrm{C}$ every $4 \mathrm{~h}$ until it reached $19 \pm 1{ }^{\circ} \mathrm{C}$, which is the optimal temperature for the development of $P$. berghei in the mosquito [35]. The mosquitoes were given access to a $6 \%$ sugar solution throughout the experiment except for the day before the blood meal.

In each experiment half of the 3-4 days old females were blood-fed on gametocytic mice, the other half were blood-fed on uninfected mice. Mice were anesthetized with an intraperitoneal injection of $8.5 \mathrm{ml} / \mathrm{kg}$ of a mix of Xylazine Xylasol ${ }^{\circledR}$ (solution: $20 \mathrm{mg} / \mathrm{ml}$ ), Ketamine Ketasol $^{\circledR}$ (solution: $100 \mathrm{mg} / \mathrm{ml}$ ) and PBS [36]. They were then placed on the top of the cages and mosquitoes were given the opportunity to blood-feed for $20 \mathrm{~min}$. One day after feeding, the females that were not completely engorged were removed from the cages. The mosquitoes were then maintained at $19{ }^{\circ} \mathrm{C}$ and $70 \%$ humidity and 
with constant access to a $6 \%$ solution of sugar up to the tests.

\section{Test for irritancy}

Irritancy of the nets was measured for 40 infected and 39 uninfected mosquitoes 11 days after blood feeding (when infected mosquitoes harboured oocysts) and for 72 infected and 64 uninfected mosquitoes 21 days after blood feeding (when most of them harboured sporozoites). The mosquitoes were placed individually into the resting part of WHO test tubes [37]. After 2 min of acclimatization, the mosquitoes were gently blown into the exposure part of the tube containing a $0.75 \%$ permethrinimpregnated paper (WHO standard paper). The time until their first jump (the time between landing on the paper and flying in an attempt to escape from the paper [38]) and the number of times they jumped between their first jump and 2 min were then recorded. This gives two independent measures of irritancy. Note that the number of jumps was measured only from the time of the first jump onwards, so that this measure of irritancy would be independent of the first. The mosquitoes were then frozen at $-20^{\circ} \mathrm{C}$ until further analysis.

\section{Test for motivation to blood feed}

Seventy-two infected and 80 uninfected mosquitoes were tested 11 days after feeding (when infected mosquitoes harboured oocysts), and 96 infectious and 107 uninfected mosquitoes were tested 21 days after feeding (when they harboured sporozoites). A third of the mosquitoes in each group were exposed either to a control paper (PY control), to $0.75 \%$ permethrin-impregnated paper for $1 \mathrm{~min}$ or to the permethrin for $2 \mathrm{~min}$. They were placed in groups of six into the resting tube of the WHO susceptibility test tubes. After $2 \mathrm{~min}$, the mosquitoes were gently blown into the test tube. After their exposure, the mosquitoes were transferred individually into $120-\mathrm{ml}$ plastic cups and given the opportunity to feed on a piece of cotton soaked with a $6 \%$ sugar solution.

To measure the mosquitoes' motivation to blood feed, $\mathrm{GH}$ placed one of his arms onto each mosquito's cup repeatedly $(2,9,24,30,36$, and $48 \mathrm{~h}$ after exposure to the insecticide) until it responded and tried to bite. The mortality of mosquitoes during the trials was recorded. All mosquitoes were frozen after the trials and kept at $-20^{\circ} \mathrm{C}$ for further analysis.

\section{Detection of Plasmodium berghei infection Oocyst detection}

To detect the oocysts, 11-day old mosquitoes were dissected in $10 \mu \mathrm{l}$ of a $0.9 \% \mathrm{NaCl}$ solution and the midguts were examined under a dissecting microscope with a fluorescent filter for the GFP. Infection was recorded as the presence or absence of oocysts.

\section{Sporozoite detection}

Sporozoites were detected with a PCR of the head and thorax of the 21-day old mosquitoes as described by Thiévent and coworkers [31]. DNA was extracted with DNAzol (MRC Inc., Cinncinati, OH, USA). Head and thorax were smashed with a pellet in $200 \mu \mathrm{l}$ of DNAzol and were incubated for $20 \mathrm{~min}$ at $55{ }^{\circ} \mathrm{C}$. The solution was then centrifuged at $20,000 \times g$ for $10 \mathrm{~min} ; 170 \mu \mathrm{l}$ of the supernatant were transferred to a new fresh tube containing $3 \mu \mathrm{l}$ of PolyAcryl Carrier (MRC Inc., Cincinnati, OH, USA) and $100 \mu \mathrm{l}$ of $100 \%$ ethanol conserved at $-20{ }^{\circ} \mathrm{C}$. After a centrifugation at $15,000 \times g$ during $8 \mathrm{~min}$, ethanol was discarded. DNA was washed with $0.8 \mathrm{ml}$ of $75 \%$ ethanol $\left(-20^{\circ} \mathrm{C}\right)$ and the tube was centrifuged $5 \mathrm{~min}$ at $15,000 \times g$. Ethanol was discarded and the pellet of DNA was dried in a Speedvac for $15 \mathrm{~min}$ at $45^{\circ} \mathrm{C}$. Finally, the DNA was eluted in $50 \mu \mathrm{l}$ of de-ionized water.

PCR amplification was done with a T3000 thermocycler (Analytik Jena AG, Jena, Germany) with $3 \mu$ of DNA and $1 \mu \mathrm{l}$ of a $10 \mu \mathrm{M}$ solution of each primer [forward (5'ACGATGATATAGATCAAAT-3 ${ }^{\prime}$ ) and reverse $\left(5^{\prime}\right.$-TAC CTAAGCTTCTTGCGTA- $3^{\prime}$ )]. Primers amplify a $111 \mathrm{bp}$ sequence of the merozoite surface protein-1 (MSP-1) gene of $P$. berghei NK65 and ANKA. Amplification was done during 40 cycles with denaturation at $95^{\circ} \mathrm{C}$, annealing at $54{ }^{\circ} \mathrm{C}$ and extension at $72{ }^{\circ} \mathrm{C}$, each for $45 \mathrm{~s}$. Infection was visually confirmed by the presence/absence of the correct band of DNA by gel electrophoresis with a $2 \%$ agarose gel containing $9 \mu \mathrm{l}$ of RedSafeTM Nucleic Acid stain (iNtRON Biotechnology, Korea).

\section{Body size}

The body size of mosquitoes was assayed as their wing length. Wings were placed onto a slide, photographed, and measured with the software ImageJ. The length of each wing was measured from the distal end of the alula to the tip of the wing (the end of the vein R3) without the fringes.

\section{Statistical methods}

The contact irritancy of permethrin was assessed by analysing: (i) the time it took mosquitoes to jump off of the insecticide-treated filter paper; and, (ii) the jumping rate, which is the number of jumps per the remaining time after their first jump (Additional file 1: Table S1). The time until first jump was analysed with a survival analysis with a mixed effect Cox model (coxme library in $\mathrm{R}$ [39]). The jumping rate was log-transformed, so that the distribution was Gaussian, and was analysed with a linear mixed model. Both analyses included the infection 
status (infected or not) and the time after infection (11 or 21 days) as nominal factors, wing length as a covariate and the mouse on which the mosquito had blood fed as a random factor.

The measure of motivation to bite was the time it took for mosquitoes to start biting after being exposed to permethrin. Because of the design (e.g., the irregular sampling points of 2, 9, 12, 24, 30, 36, $48 \mathrm{~h}$ after exposure), an interval count analysis was used instead of a more classical survival analysis (see [40]). Since the data followed neither an exponential nor a Weibull distribution, a cox-hazard survival analysis was approximated by analysing the number of mosquitoes feeding at a given time as a function of the interval of time between two measurements [40] (Additional file 1: Table S2). The exposure (permethrin or control), the infection status (infected or not), the time after infection (11 or 21 days) were added as nominal factors, and wing length as a covariate. To obtain the results shown below, non-significant interactions were removed from the best model (with p-values greater than 0.25). Significance of the effects were assessed using a type 3 anova (car library in R [41]).

All analyses and graphs were done with the software $\mathrm{R}$ (version 3.4.3) [42] with the Rstudio interface (version 1.1.419) [43]. Mixed models (LMM and GLMM) were done with the lme 4 library in R [44].

\section{Results}

For the irritancy experiment, $69.6 \%$ of the mosquitoes that blood-fed on gametocytic mice were infected, while for the sub-lethal effects experiment, $84.5 \%$ of them were infected. The mosquitoes that had fed on infected mice but did not harbour parasites were removed from the analysis. In total, the behaviour of 510 mosquitoes was analysed, of which 88 harboured oocysts, 132 harboured sporozoites and 290 were uninfected.

\section{Irritancy}

Eleven days after infection irritancy was measured for 26 oocyst-infected and 39 uninfected mosquitoes; 21 days after infection it was measured for 52 sporozoiteinfected mosquitoes and 64 uninfected ones. On average, it took about $24.3 \mathrm{~s}$ for mosquitoes to jump off of the insecticide-treated paper (Fig. 1), and they jumped about 4.8 times per minute. Neither the time up to the first jump $\left(\mathrm{x}^{2}=1.73, \mathrm{df}=1, \mathrm{p}=0.19\right)$ nor the jumping rate $\left(\mathrm{x}^{2}=1.08, \mathrm{df}=1, \mathrm{p}=0.3\right)$ differed significantly between infected and uninfected mosquitoes and neither the time up to the first jump $\left(x^{2}=1.19, \mathrm{df}=1, \mathrm{p}=0.28\right)$ nor the jumping rate ( $\left.L M M, \chi^{2}=0.08, p=0.78\right)$ was linked to the age of the mosquitoes. The interaction between the infection status and the time after infection had no significant effect on the time up to the first jump $\left(x^{2}=0.01, \mathrm{df}=1\right.$, $\mathrm{p}=0.93)$ or the jumping rate $\left(\mathrm{x}^{2}=0.06, \mathrm{df}=1, \mathrm{p}=0.3\right)$. Finally, wing length had no effect on either time up to the first jump $\left(\mathrm{x}^{2}=0.37, \mathrm{df}=1, \mathrm{p}=0.54\right)$ or the jumping rate $\left(\mathrm{x}^{2}=0.03, \mathrm{df}=1, \mathrm{p}=0.87\right)$.

\section{Blood feeding motivation after exposition to permethrin} None of the mosquitoes died during the trials, i.e., within the $48 \mathrm{~h}$ following exposure to the insecticidetreated or untreated paper. The motivation to feed of 329
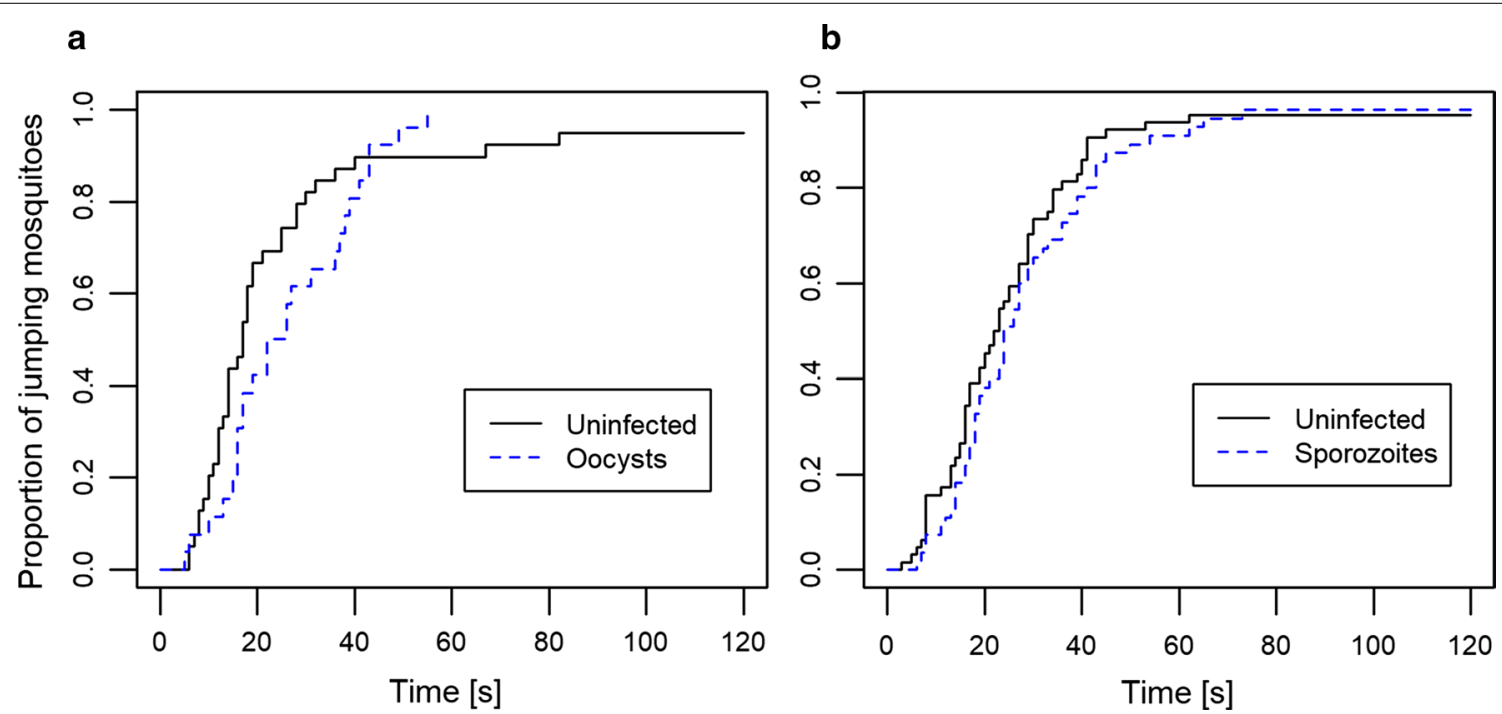

Fig. 1 Cumulative proportion of jumping mosquitoes as a function of time since exposure. a Proportion measured 11 days after blood feeding, when infected mosquitoes were harbouring oocysts. b Proportion measured 21 days after infection, when infected mosquitoes were carrying sporozoites in their salivary glands 
mosquitoes was analysed. Of the 142 tested 11 days after infection, 62 were infected with oocysts and the remainder were uninfected. Of the 187 tested 21 days after infection, 80 were infected with sporozoites.

The summary of the statistical analysis of the proportion of mosquitoes that tried to bite at each test (which, for simplicity, will be called biting rate) is shown in Table 1. The main results are the following. First, if the mosquitoes had been exposed to the control paper, $88.5 \%$ of them tried to bite within $24 \mathrm{~h}$ after exposure, and their biting rate decreased with time (Fig. 2). If they had been exposed to permethrin, fewer mosquitoes tried to bite

Table 1 Interval count analysis of the motivation to bite of mosquitoes after a previous permethrin or control exposure in function of time

\begin{tabular}{lccc}
\hline Factors & $\mathbf{X}^{\mathbf{2}}$ & Df & $\mathbf{p}$-value \\
\hline Infection status & 0.97 & 1 & 0.32 \\
Type of exposure & 103.24 & 2 & $<0.001$ \\
Age & 0.01 & 1 & 0.92 \\
Time & 34.81 & 5 & $<0.001$ \\
Infection status: time & 11.8 & 5 & 0.04 \\
Type of exposure: age & 9.1 & 2 & 0.01 \\
Type of exposure: time & 99.16 & 10 & $<0.001$ \\
Age: time & 14.16 & 5 & 0.01 \\
\hline
\end{tabular}

within $24 \mathrm{~h}$ after exposure ( 1 min exposure: $57.5 \%$; $2 \mathrm{~min}$ exposure: $50.5 \%)$ and their biting rate increased with the time.

Second, infected mosquitoes tried to bite earlier than uninfected mosquitoes. Thus, 11 days after infection $81.5 \%$ of the oocyst-infected mosquitoes tried to bite within $24 \mathrm{~h}$ after being exposed to permethrin, while only $57.1 \%$ of the uninfected ones did, and 21 days after infection $73.6 \%$ of the sporozoite-infected mosquitoes tried to bite $24 \mathrm{~h}$ after exposure while only $49.8 \%$ of the uninfected ones did. The statistical analysis (Table 1) confirms that the biting rate decreased more rapidly with time for uninfected mosquitoes than for infected ones. This difference, however, was not affected by the age of the mosquito (so, by the stage of the parasite) (infection status "age "time: $\left.\chi^{2}=0.2, \mathrm{df}=5, \mathrm{p}=0.65\right)$ or by the type of exposure (infection status" exposure ${ }^{*}$ time: $x^{2}=5.98$, $\mathrm{df}=12, \mathrm{p}=0.91$ ). (Note that these two interactions were not included in the final model shown in Table 1).

Third, the young mosquitoes (11 days after their blood meal) attempted to bite earlier than old mosquitoes ( 21 days after their first blood meal): 24 h post exposition, $69.3 \%$ of the young mosquitoes had tried to bite while $61.7 \%$ of the old ones had. In addition, the effect of the exposure to permethrin was stronger for old mosquitoes than for young ones. Thus, the time it took to young mosquitoes to try to bite was similar whether they had been
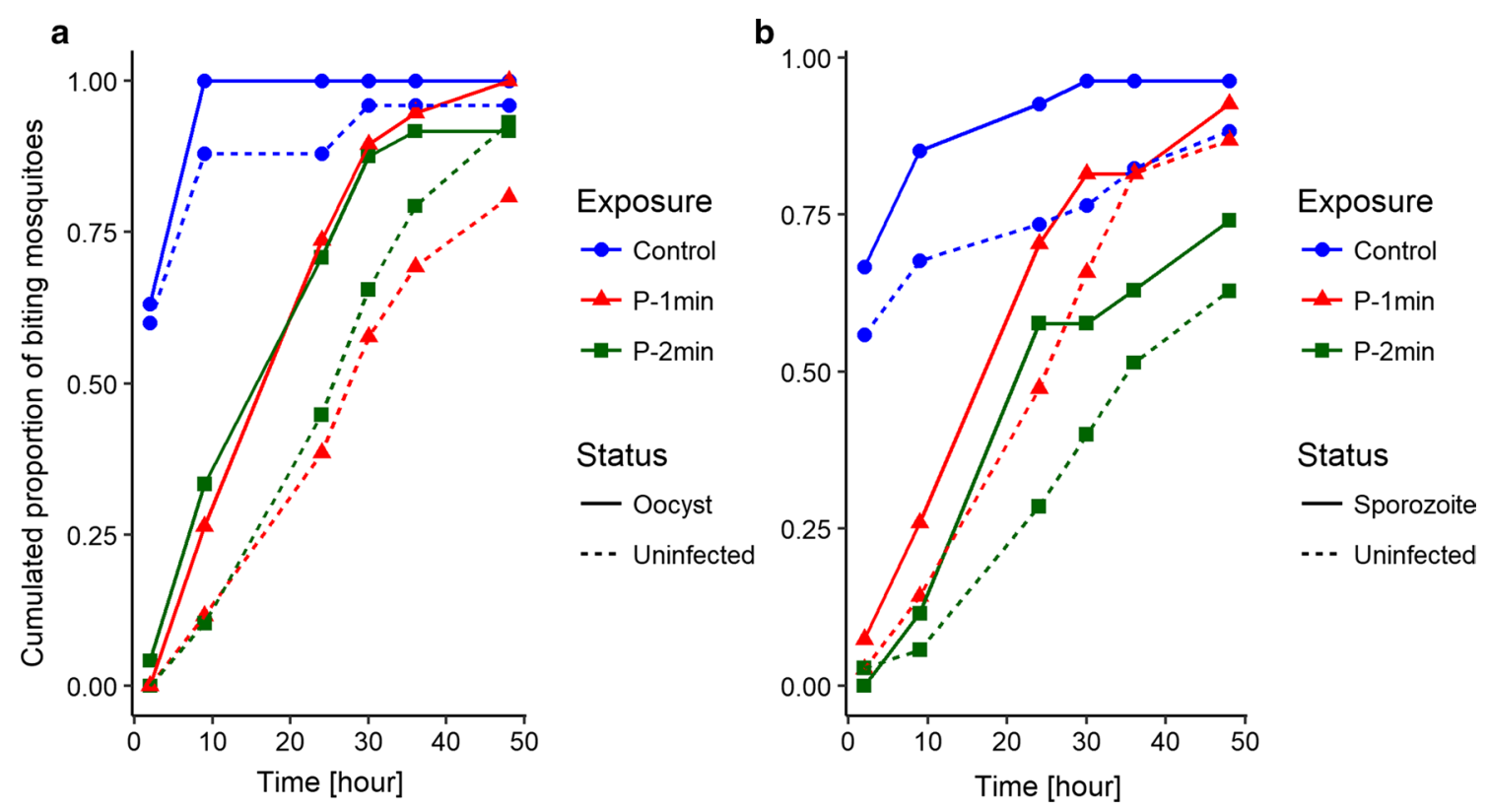

Fig. 2 Cumulated proportion of biting mosquitoes in function of time. Colours designate the type of exposure: blue dots denote exposure on control paper; red triangles denote exposure to permethrin for $1 \mathrm{~min}$; green squares denote exposure for $2 \mathrm{~min}$. Continuous lines represent the infected mosquitoes and the dashed lines represent the uninfected ones. a Cumulated proportion of biting mosquitoes tested 11 days after infection (i.e., infected with oocysts). b Cumulated proportion of biting mosquitoes tested 21 days after infection (i.e., infected with sporozoites) 
exposed to permethrin for 1 or for 2 min (Fig. 2a). In the older mosquitoes, however, the mosquitoes exposed to permethrin for $1 \mathrm{~min}$ attempted to bite earlier than those exposed for 2 min (Fig. 2b).

\section{Discussion}

One of the ways ITNs protect their users is that the insecticide irritates mosquitoes when they touch it, so that they are less likely to pass through the net to bite. Since mosquitoes can only be irritated if they are exposed to some insecticide, irritancy can lead to sub-lethal effects of insecticide. Indeed, in this experiment a short exposure to permethrin made mosquitoes less likely to try to bite for almost $48 \mathrm{~h}$ without inducing any mortality. While irritancy was not affected by the infection by the malaria parasite, the inhibition of biting was shorter for malaria-infected mosquitoes than for uninfected ones.

The intensity or duration of sub-lethal exposure for mosquitoes depends mainly on how strongly they are irritated. Here the irritancy (measured in two ways: time until first jump and jumping rate [38]) was similar for malaria-infected and for uninfected mosquitoes. This suggests that when facing an ITN, infected and uninfected mosquitoes would receive the same sub-lethal dose of insecticide. It should, however, be noted that the design used here did not allow to measure the persistence of mosquitoes that try to bite through an ITN, which would give a more complete idea about the exposure to the insecticide.

Sub-lethal exposure to insecticides affects the life history traits of insects in various ways $[6,7]$, including that pyrethroids affects their feeding capacities [18-23]. This study corroborated these results by showing that a short exposure to permethrin (which induced no mortality) alters the mosquito's host-seeking behaviour. Indeed, the feeding motivation of mosquitoes exposed to permethrin for 1 or 2 min was inhibited for almost $48 \mathrm{~h}$.

More surprising is that this inhibition was weaker for malaria-infected mosquitoes than for uninfected ones. Indeed, our original idea was that infectious mosquitoes (so, those with sporozoites) would be less inhibited and start biting earlier than uninfected ones, for they are more motivated to bite than uninfected or oocyst-infected (and thus non-infectious) ones [26-30]. However, that oocyst-infected mosquitoes were also less inhibited than uninfected ones does not support this idea. Indeed, the manipulation by oocysts (non-infectious stage) should go in the opposite direction: to increase its transmission, the parasite should preserve mosquitoes from host defenceassociated mortality, and therefore decrease their motivation to bite [28].

An alternative explanation for the weaker effect of permethrin on infected mosquitoes may be linked to oxidative stress. Indeed, the production of reactive oxygen species (ROS) is increased by both infection by malaria [45-48] and pyrethroid exposure [49, 50]. After an infected blood meal, mosquitoes modulate this ROS production by expressing a higher level of antioxidant enzymes in the fat body [45]. Similarly, survival to pyrethroid exposure requires sufficient activity of anti-oxidant enzymes to avoid lipid peroxidation and cell damage [51]. It is therefore possible that the higher anti-oxidant defence triggered by an infection with Plasmodium may, to some extent, help to deal with a further exposure to a pyrethroid insecticide. Furthermore, detoxification mechanisms may also play a role. It is known for example that following infection by Plasmodium, the cytochrome P450 CYP6M2, an enzyme involved in pyrethroid's detoxification [52, 53], is upregulated [54]. Thus, because of the higher production of both anti-oxidant and cytochrome P450 enzymes, it is not worth to expect infected mosquitoes to recover faster after permethrin exposure. This could help to explain why, in this study, they started to probe sooner after exposure. Such a link would have epidemiological implications, for it implies that infected mosquitoes would be more tolerant to pyrethroids. Glunt and coworkers [55] investigated this link, and reported no effect of $P$. yoelii sporozoite-infection on An. stephensi resistance to permethrin, but slightly higher knockdown resistance for mosquitoes that had fed on infectious blood than for those fed on uninfected blood. However, as they mentioned, some of the mosquitoes that had fed on infectious blood may not have been infected, possibly hiding any greater effect of malaria infection on permethrin sensitivity.

A further look into the different mechanisms implied in pyrethroid metabolism may also help to explain the longer inhibition of blood feeding behaviour in older mosquitoes. A possibility may be linked to the fact that resistance to insecticides often decreases with age (e.g., $[34,56,57])$, which is due in part to the age-related decline of the activity of P450 mono-oxygenases [58], esterases [59] and glutathione-S-transferases [56], the three main enzymatic systems responsible for pyrethroid resistance in mosquitoes [60,61]. Old mosquitoes may thus need more time to detoxify and recover after a pyrethroid exposure. This idea would be consistent with the observation of this study: that old mosquitoes responded differently to the duration of exposure (1 or $2 \mathrm{~min}$ ), whereas young mosquitoes showed a similar inhibition response for the two exposures.

Whatever the mechanisms, the additional inhibition of mosquito's host-seeking behaviour induced by permethrin may have strong implications for the epidemiology of malaria. Indeed, each time a mosquito is exposed to a sublethal dose of permethrin, it will postpone its blood meal 
and thus might lengthen its gonotrophic cycle [45]. This would strongly affect not only the mosquito's reproductive success, but also the transmission of malaria. First, lower biting rate of uninfected mosquitoes further than what is expected from the physical barrier and repellency of ITNs would further decrease the rate of encountering malaria. Second, the lower biting rate of oocyst-infected mosquitoes would increase the chance that oocyst-infected mosquitoes would survive to harbour sporozoites. Third, the inhibition of the infectious mosquitoes would reduce the rate at which sporozoites are transmitted. Since, however, that the infection reduces the inhibition of biting, it also reduces the impact of the sub-lethal exposure on the epidemiology of malaria through the two latter effects.

Whether such effects are indeed observed depends on whether we see similar patterns in a natural system. Furthermore, since some effects of inhibition can increase transmission while others decrease it, these results should be confirmed not only qualitatively, but also quantitatively with a natural human malaria parasite in different epidemiological settings.

\section{Conclusions}

ITNs offer personal protection in part by irritating mosquitoes and thereby reducing the probability to being bitten by an infectious mosquito. Here, irritancy had an additional effect than just reducing the time mosquitoes spend on the net trying to bite or pass through it. Indeed, sub-lethal exposure (1 or $2 \mathrm{~min}$ ) to permethrin, inhibited the host-seeking behaviour of mosquitoes by impeding them to blood feed for almost $48 \mathrm{~h}$, confirming the results of previous studies [22, 23]. In addition, the results of this study complement a study showing that the sporozoites of malaria reduce the repellency of an insecticide [31]. Thus, malaria infection shortened the inhibition caused by the insecticide at, both, its developmental (oocyst) and infectious (sporozoite) stages. The results of this study may help to get a better measure of the impact of ITNs for malaria control. On the one hand, the inhibition of the mosquito's host-seeking behaviour might reduce the chance they encounter and/or transmit malaria, thus increasing not only the protection of the ITNs users, but also the protection of the non-users. On the other hand, by shortening the inhibition of mosquito feeding behaviour, both oocysts and sporozoites might weaken both personal and community protections.

\section{Additional file}

Additional file 1: Table S1. Dataset Irritancy. Data used for statistical analysis of irritancy. Table S2. Dataset Motivation to bite. Data used for statistical analysis of the motivation of mosquitoes to bite after permethrin exposure (interval count analysis).
Authors' contributions

All authors conceived and designed the study. JCK coordinated the study. KT, $\mathrm{GH}$ and OE performed the lab work and collected the data. KT, GH and JCK carried out the statistical analysis. KT, GH and JCK wrote the original draft and all authors contributed to the elaboration of the final version of the manuscript. All authors read and approved the final manuscript.

\section{Acknowledgements}

We thank Volker Heussler's team for providing the mice and the malaria parasites, Olivier Rais for dealing with the mice during infection, and Pietro Storelli and Noémie loset for their help during data collection.

\section{Competing interests}

The authors declare that they have no competing interests.

\section{Consent for publication}

Not applicable.

Data availability statement

The datasets analysed during the current study are included in this published article (and its Additional file).

\section{Ethics approval and consent to participate}

All procedures that involved mice were conducted in accordance with the guidelines of the Swiss Tierschutzgesetz (Animal Rights Laws) and approved by the ethical committee of the University of Bern (Permit Number: BE109/13).

Funding

Not applicable.

\section{Publisher's Note}

Springer Nature remains neutral with regard to jurisdictional claims in published maps and institutional affiliations.

Received: 21 December 2018 Accepted: 11 March 2019

Published online: 14 March 2019

\section{References}

1. Lengeler C. Insecticide-treated bed nets and curtains for preventing malaria. Cochrane Database Syst Rev. 2004;2:CD000363.

2. Birget PLG, Koella JC. A genetic model of the effects of insecticide-treated bed nets on the evolution of insecticide resistance. Evol Med Public Health. 2015;2015:205-15.

3. Chareonviriyaphap T. Behavioral responses of mosquitoes to insecticides. In: Perveen FK, editor. Insecticides: pest engineering. Croatia: Intech Open Access Publisher; 2012

4. Curtis CF, Lines JD, ljumba J, Callaghan A, Hill N, Karimzad MA. The relative efficacy of repellents against mosquito vectors of disease. Med Vet Entomol. 1987;1:109-19.

5. Dusfour I, Achee NL, Roberts DR, Grieco JP. Contact irritancy and spatial repellency behaviors in Anopheles albimanus Wiedemann (Diptera: Culicidae) collected in Orange Walk, Belize, CA. J Vector Ecol. 2009:34:232-7.

6. Lee C-Y. Sublethal effects of insecticides on longevity, fecundity and behaviour of insect pests: a review. J Biosci. 2000;11:107-12.

7. Desneux N, Decourtye A, Delpuech J-M. The sublethal effects of pesticides on beneficial arthropods. Annu Rev Entomol. 2007:52:81-106.

8. Haynes KF. Sublethal effects of neurotoxic insecticides on insect behavior. Annu Rev Entomol. 1988;33:149-68.

9. Robert LL, Olson JK. Effects of sublethal dosages of insecticides on Culex quinquefasciatus. J Am Mosq Control Assoc. 1989;5:239-46.

10. Reyes-Villanueva F, Juarez-Eguia M, Flores-Leal A. Effects of sublethal dosages of Abate upon adult fecundity and longevity of Aedes aegypti. J Am Mosq Control Assoc. 1990;6:739-41.

11. Flores AE, Garcia GP, Badii MH, Rodriguez Tovar MAL, Fernandez Salas I. Effects of sublethal concentrations of Vectobac on biological parameters of Aedes aegypti. J Am Mosq Control Assoc. 2004;20:412-7. 
12. Muturi EJ, Lampman R, Costanzo K, Alto BW. Effect of temperature and insecticide stress on life-history traits of Culex restuans and Aedes albopictus (Diptera: Culicidae). J Med Entomol. 2011;48:243-50.

13. Sanil D, Shetty NJ. The effect of sublethal exposure to temephos and propoxur on reproductive fitness and its influence on circadian rhythms of pupation and adult emergence in Anopheles stephensi Liston-a malaria vector. Parasitol Res. 2012;111:423-32.

14. Yadav P, Barde PV, Gokhale MD, Vipat V, Mishra AC, Pal JK, Mourya DT. Effect of temperature and insecticide stresses on Aedes aegypti larvae and their influence on the susceptibility of mosquitoes to dengue-2 virus. Southeast Asian J Trop Med Public Health. 2005;36:1139-44.

15. Muturi EJ, Costanzo K, Kesavaraju B, Alto BW. Can pesticides and larva competition alter susceptibility of Aedes mosquitoes (Diptera: Culicidae) to arbovirus infection? J Med Entomol. 2011:48:429-36.

16. Muturi EJ, Alto BW. Larval environmental temperature and insecticide exposure alter Aedes aegypti competence for arboviruses. Vector Borne Zoonotic Dis. 2011;11:1157-63.

17. Vantaux A, Ouattarra I, Lefèvre T, Dabiré KR. Effects of larvicidal and larval nutritional stresses on Anopheles gambiae development, survival and competence for Plasmodium falciparum. Parasites Vectors. 2016;9:226.

18. Kwan WH, Gatehouse AG. The effects of low doses of three insecticides on activity, feeding, mating, reproductive performance and survival in Glossina morsitans morsitans (Glossinidae). Entomol Exp Appl. 1978;23:201-21.

19. Chadd EM, Brady J. Sublethal insecticide effects on the probing responsiveness of tsetse flies and blowflies. Physiol Entomol. 1982;7:133-41.

20. Hall FR, Thacker JRM. Laboratory studies on effects of three permethrin formulations on mortality, fecundity, feeding, and repellency of the twospotted spider mite (Acari: Tetranychidae). J Econ Entomol. 1993;86:537-43.

21. Armstrong KF, Bonner AB. Investigation of a permethrin-induced antifeedant effect in Drosophila melanogaster: an ethological approach. Pestic Sci. 1985;16:641-50.

22. Liu W, Todd RG, Gerberg EJ. Effect of three pyrethroids on blood feeding and fecundity of Aedes aegypti. J Am Mosq Control Assoc. 1986:2:310-3.

23. Glunt KD, Coetzee M, Huijben S, Koffi AA, Lynch PA, N'Guessan R, et al. Empirical and theoretical investigation into the potential impacts of insecticide resistance on the effectiveness of insecticide-treated bed nets. Evol Appl. 2018;11:431-41.

24. Cohnstaedt LW, Allan SA. Effects of sublethal pyrethroid exposure on the host-seeking behavior of female mosquitoes. J Vector Ecol. 2011;36:395-403.

25. Spitzen J, Koelewijn T, Mukabana WR, Takken W. Effect of insecticide-treated bed nets on house-entry by malaria mosquitoes: the flight response recorded in a semi-field study in Kenya. Acta Trop. 2017;172:180-5.

26. Koella JC, Sørensen FL, Anderson RA. The malaria parasite, Plasmodium falciparum, increases the frequency of multiple feeding of its mosquito vector, Anopheles gambiae. Proc R Soc Lond B Biol Sci. 1998;265:63-8.

27. Wekesa JW, Copeland RS, Mwangi RW. Effect of Plasmodium falciparum on blood feeding behavior of naturally infected Anopheles mosquitoes in western Kenya. Am J Trop Med Hyg. 1992;47:484-8.

28. Koella JC, Rieu L, Paul REL. Stage-specific manipulation of a mosquito's host-seeking behavior by the malaria parasite Plasmodium gallinaceum. Behav Ecol. 2002:13:816-20.

29. Anderson RA, Koella JC, Hurd H. The effect of Plasmodium yoelii nigeriensis infection on the feeding persistence of Anopheles stephensi Liston throughout the sporogonic cycle. Proc R Soc Lond B Biol Sci. 1999;266:1729-33.

30. Rossignol PA, Ribeiro JM, Spielman A. Increased biting rate and reduced fertility in sporozoite-infected mosquitoes. Am J Trop Med Hyg. 1986;35:277-9.

31. Thiévent K, Hofer L, Rapp E, Tambwe MM, Moore S, Koella JC. Malaria infection in mosquitoes decreases the personal protection offered by permethrin-treated bednets. Parasites Vectors. 2018;11:284.

32. Viana M, Hughes A, Matthiopoulos J, Ranson H, Ferguson HM. Delayed mortality effects cut the malaria transmission potential of insecticideresistant mosquitoes. Proc Natl Acad Sci USA. 2016;113:8975-80.
33. WHO. Global insecticide use for vector-borne disease control. 4 th ed. Geneva: World Health Organization; 2009.

34. Kulma K, Saddler A, Koella JC. Effects of age and larval nutrition on phenotypic expression of insecticide-resistance in Anopheles mosquitoes. PLoS ONE. 2013;8:e58322.

35. Vanderberg JP. Plasmodium berghei: quantitation of sporozoites injected by mosquitoes feeding on a rodent host. Exp Parasitol. 1977;42:169-81.

36. Wolfensohn S, Lloyd M. Handbook of laboratory animal management and welfare. 4th ed. New Jersey: Wiley; 2013.

37. WHO. Test procedures: for insecticide resistance monitoring in malaria vector mosquitoes. Geneva: World Health Organization; 2013.

38. Evans RG. Laboratory evaluation of the irritancy of bendiocarb, lambda-cyhalothrin and DDT to Anopheles gambiae. J Am Mosq Control Assoc. 1993;9:285-93.

39. Therneau T. Mixed effects Cox models. 2015; CRAN Repos.

40. Egli P, Schmid B. The analysis of complex leaf survival data. Basic Appl Ecol. 2001;2:223-31.

41. Fox J, Weisberg S. An R companion to applied regression. SAGE: LoS Angeles; 2010.

42. R Core Team. R: a language and environment for statistical computing. 2013.

43. R Studio Team. R Studio: integrated development environment for R. 2016.

44. Bates D, Maechler M, Bolker B, Walker S. Fitting linear mixed-effects models using Ime4. J Stat Softw. 2015;67:1-48.

45. Molina-Cruz A, DeJong RJ, Charles B, Gupta L, Kumar S, JaramilloGutierrez G, et al. Reactive oxygen species modulate Anopheles gambiae immunity against bacteria and Plasmodium. J Biol Chem. 2008;283:3217-23.

46. Kumar S, Christophides GK, Cantera R, Charles B, Han YS, Meister $\mathrm{S}$, et al. The role of reactive oxygen species on Plasmodium melanotic encapsulation in Anopheles gambiae. Proc Natl Acad Sci USA. 2003;100:14139-44.

47. Kumar S, Gupta L, Han YS, Barillas-Mury C. Inducible peroxidases mediate nitration of Anopheles midgut cells undergoing apoptosis in response to Plasmodium invasion. J Biol Chem. 2004;279:53475-82

48. Chaitanya R, Sridevi P, Kumar KS, Mastan BS, Kumar KA, Dutta-Gupta A. Expression analysis of reactive oxygen species detoxifying enzyme genes in Anopheles stephensi during Plasmodium berghei midgut invasion. Asian Pac J Trop Med. 2014;7:680-4.

49. Müller P, Chouaïbou M, Pignatelli P, Etang J, Walker ED, Donnelly MJ, et al. Pyrethroid tolerance is associated with elevated expression of antioxidants and agricultural practice in Anopheles arabiensis sampled from an area of cotton fields in Northern Cameroon. Mol Ecol. 2008:17:1145-55.

50. Abdollahi M, Ranjbar A, Shadnia S, Nikfar S, Rezaie A. Pesticides and oxidative stress: a review. Med Sci Monit. 2004;10:141-7.

51. Vontas J, Small GJ, Hemingway J. Glutathione S-transferases as antioxidant defence agents confer pyrethroid resistance in Nilaparvata lugens. Biochem J. 2001;357(Pt 1):65-72.

52. Stevenson BJ, Bibby J, Pignatelli P, Muangnoicharoen S, O'Neill PM, Lian LY, et al. Cytochrome P450 6M2 from the malaria vector Anopheles gambiae metabolizes pyrethroids: sequential metabolism of deltamethrin revealed. Insect Biochem Mol Biol. 2011;41:492-502.

53. Feyereisen R. Insect CYP genes and P450 enzymes. Insect Mol Biol Biochem. 2012. https://doi.org/10.1016/B978-0-12-384747-8.10008-X.

54. Félix RC, Müller P, Ribeiro V, Ranson H, Silveira H. Plasmodium infection alters Anopheles gambiae detoxification gene expression. BMC Genom. 2010;11:312.

55. Glunt KD, Thomas MB, Read AF. The effects of age, exposure history and malaria infection on the susceptibility of Anopheles mosquitoes to low concentrations of pyrethroid. PLoS ONE. 2011:6:e24968.

56. Rajatileka S, Burhani J, Ranson H. Mosquito age and susceptibility to insecticides. Trans R Soc Trop Med Hyg. 2011;105:247-53.

57. Aïzoun N, Aïkpon R, Azondekon R, Asidi A, Akogbéto M. Comparative susceptibility to permethrin of two Anopheles gambiae s.l. populations from Southern Benin, regarding mosquito sex, physiological status, and mosquito age. Asian Pac J Trop Biomed. 2014;4:312-7. 
58. Xu T-L, Zhong D-B, Fang Q, Tang L-H, Zhou S-S, Zheng B. Changes of pyrethroid resistance and P450 monooxygenase activity with age in Anopheles sinensis in Huainan City, Anhui Province, China. Zhongguo Xue Xi Chong Bing Fang Zhi Za Zhi. 2013;25(157-9):166 (in Chinese)

59. Dahan-Moss YL, Koekemoer LL. Analysis of esterase enzyme activity in adults of the major malaria vector Anopheles funestus. Parasites Vectors. 2016;9:110.
60. Hemingway J, Hawkes NJ, McCarroll L, Ranson H. The molecular basis of insecticide resistance in mosquitoes. Insect Biochem Mol Biol. 2004;34:653-65.

61. Nkya TE, Akhouayri I, Kisinza W, David J-P. Impact of environment on mosquito response to pyrethroid insecticides: facts, evidences and prospects. Insect Biochem Mol Biol. 2013;43:407-16.
Ready to submit your research? Choose BMC and benefit from:

- fast, convenient online submission

- thorough peer review by experienced researchers in your field

- rapid publication on acceptance

- support for research data, including large and complex data types

- gold Open Access which fosters wider collaboration and increased citations

- maximum visibility for your research: over $100 \mathrm{M}$ website views per year

At BMC, research is always in progress.

Learn more biomedcentral.com/submissions 